\title{
Development of an advanced injection time model for an autoinjector
}

This article was published in the following Dove Press journal:

Medical Devices: Evidence and Research

\section{Thomas Thueer \\ Lena Birkhaeuer \\ Declan Reilly}

Device Development, Pharma Technical Development Europe, F. Hoffmann-La Roche Ltd, Basel, Switzerland
Correspondence: Thomas Thueer F. Hoffmann-La Roche Ltd, B9/7II.3, 4070 Basel, Switzerland

Tel +4I 616880045

Email thomas.thueer@roche.com
Background: This work describes an advanced physics-based mathematical model that accurately predicts autoinjector injection time. Autoinjectors are a well-established technology for parenteral drug delivery and quantifying the probability to achieve a given injection time is critical to the successful development and commercial launch of the autoinjector.

Method: Each parameter that can influence injection time was treated as a statistical variable with an appropriate distribution function. Monte Carlo simulation was used to obtain the probability of achieving the required injection time. Sensitivity analyses were performed to identify those parameters most critical in contributing to the overall injection time. To validate the model, a number of experiments were conducted on autoinjectors, with key contributors to injection time measured and characterized.

Results: The results showed excellent agreement between modeled and measured injection time. The modeling error for all investigated device configurations was smaller than $12 \%$ and the error range was less than $6 \%$. The consistent over-estimation of injection time suggests a small bias in the model which could be accounted for by reducing internal friction.

Conclusion: This work provides evidence that the selected modeling approach, which aims for a simple yet computationally inexpensive model, is accurate and enables running comprehensive statistical simulations to determine the full range of expected injection times due to component variability.

Keywords: plunger force, viscosity, stopper friction, sensitivity analysis, model validation

\section{Introduction}

The development of parenteral drug delivery devices for biotechnological drugs is a challenging endeavor due to the inherent complexity of the systems. A preferred type of device for home-use subcutaneous injection is the autoinjector because of its ease of use for patients and the possibility to tailor specific features to the needs of the targeted patient group. ${ }^{1}$

One performance attribute of an autoinjector which has received significant attention is injection time, that is, the time it takes to deliver the drug content from the device, including device-to-device variation. Therefore, it is key to a successful autoinjector development to identify the main contributors to injection time early in the project and to obtain comprehensive understanding of the impact of parameter variability, as caused by variation in manufacturing of the drug product and device components or environmental conditions at point of use, on the probability of achieving the desired injection time.

This article describes a model to predict injection time of an autoinjector taking into account a large number of input parameters and their associated variability. Such 
a model is one of the tools available to developers to reliably predict the potential range of injection times and therefore keep control over this critical parameter from early phase development through to commercialization.

Previously published articles have described various aspects of injection time modeling for autoinjectors. Injection time models of different detail and complexity have been presented. One approach to calculate injection time is based on the equation of motion for the stopper which can be simplified by approximations to yield an explicit solution. ${ }^{2}$ Another approach is to model all components in a software tool (eg, Simulink $®)$ and let the software solve the equation system for injection time. ${ }^{3}$ Further models for injection time calculation are based on the equilibrium of static forces which include stopper friction and pressure drop through the needle as described by the Hagen-Poiseuille equation. ${ }^{4}$ Several publications have assessed the impact of different parameters on autoinjector performance. Variability of components as a source for injection time variation was investigated, and non-Newtonian behavior of fluids was studied in detail. ${ }^{1-6}$ Internal friction must also be considered for autoinjector performance, but it has a significantly larger impact in devices with screw thread drive systems like insulin pens. ${ }^{7}$

This article describes an advanced injection time model that follows a similar but refined modeling approach with an in-depth look at specific models for drug viscosity, plunger force and stopper friction. This work is the first to address model accuracy in a quantitative way as determined through model validation. These results, along with the assessment of component variability by means of probabilistic simulations, confirm that injection time can be predicted with high confidence.

\section{Materials and methods}

\section{Materials}

The presented injection time model was created for a threestep disposable autoinjector (Figure 1). The autoinjector com- prises a $1 \mathrm{~mL}$ pre-filled syringe with staked needle containing a monoclonal antibody drug (mAb). The viscosity of the drug is $9.4 \mathrm{mPa} \cdot \mathrm{s}$ at $20^{\circ} \mathrm{C}$ and nominal protein concentration.

Force measurements were taken on a zwickiLine Z0.5 by Zwick. Different custom fixtures were used to hold test samples in place during measurements. Computed tomography scans of the syringe needle were generated on a Zeiss Metrotom 1500. The model is implemented in Microsoft Excel and makes use of the Crystal Ball plugin by Oracle.

\section{Model overview}

The model considers static forces acting on the stopper. It is acceptable to neglect inertial terms for analysis of the drug delivery process due to low masses and velocities. The main forces that contribute to injection time are the stopper friction, the fluidic force caused by the pressure drop through the needle and the plunger force which is driving the stopper forward (Figure 2). ${ }^{8}$ Additional friction may occur in the drive system of the device. However, such friction is included in the plunger force measurement which measures the net force acting on the plunger. Effects of pressure drop across the syringe barrel or due to the constriction at the needle entry can be neglected given their order of magnitude compared to the above-listed forces. ${ }^{1}$

After setting up the equilibrium of forces (Equation 1), the equation can be solved for injection time (Equation 2).

$$
\begin{gathered}
F_{\text {plunger }}=F_{\text {friction }}+F_{\text {fluidic }} \\
t_{i n j}=f\left(\mu, L, x, R^{4}, \frac{1}{r^{4}}, \frac{1}{\left(F_{\text {plunger }}-F_{\text {friction }}\right)}\right)
\end{gathered}
$$

where $t_{i n j}$ is injection time, $\mu$ is viscosity, $L$ is needle length, $x$ is stopper displacement, $R$ is inner radius of syringe barrel, $r$ is inner radius of needle, $F_{\text {plunger }}$ is plunger force, $F_{\text {friction }}$ is

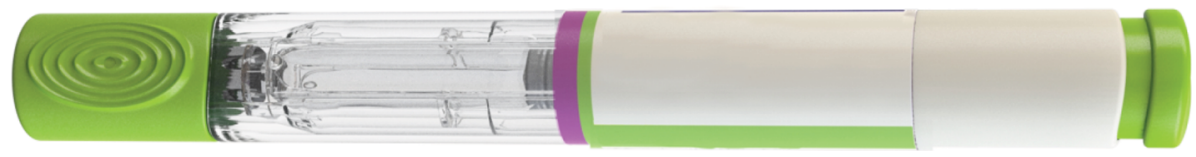

Figure I Disposable autoinjector for delivery of a monoclonal antibody drug.

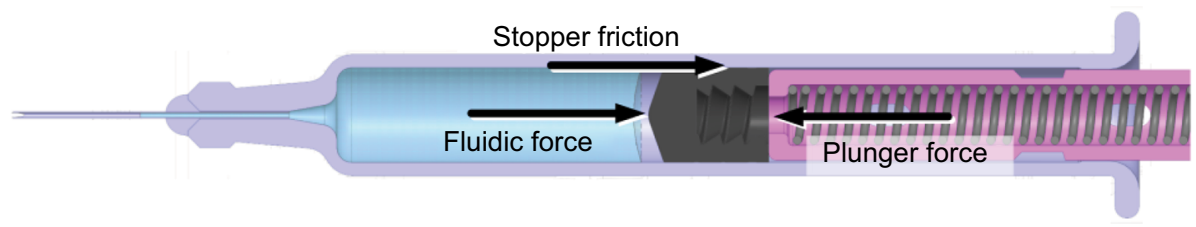

Figure 2 Schematic of the main forces contributing to injection time: stopper friction, fluidic force and plunger force. 
stopper friction and $F_{\text {fluidic }}$ is resistive force caused by the pressure drop in the needle.

Even though the model includes static forces only, the parameter time is part of the equation in order to express the flow rate $(Q)$ through the needle as part of the HagenPoiseuille law (Equation 3).

$$
\Delta p=\frac{8 \mu L Q}{\pi r^{4}}
$$

where $\Delta p$ is pressure drop, $\mu$ is viscosity (function of protein concentration and temperature), $L$ is needle length, $Q$ is volumetric flow rate and $r$ is the radius of the needle bore.

This formula assumes laminar flow which is given for the parameters of the discussed autoinjector.

The viscosity of the mAb contained in the autoinjector can be modeled with one value (for a given set of temperature and protein concentration) as the mAb is known to exhibit Newtonian behavior. For future use of the model with other drugs, approaches for modeling of non-Newtonian fluids are described in the literature. ${ }^{5,6,8}$ The use of the Newtonian model will overestimate injection time should shear thinning occur, and this is generally considered conservative as most autoinjector developments will target short injection times.

In the following sections, detailed models of selected parameters are described.

\section{Viscosity model}

The viscosity of the mAb formulation is highly dependent on protein concentration and temperature. Therefore, it is important to understand the behavior of viscosity as a function of these parameters. A viscosity model was derived for the $\mathrm{mAb}$ in the autoinjector under consideration from measurements of different formulation batches at different protein concentration levels and temperatures. A fit function was found (Equation 4) such that the logarithm of the viscosity $(\mu)$ is a function of the protein concentration $(c)$ and the inverse of the temperature $(T)$.

$$
\mu=e^{a_{1}+a_{2} * c+a_{3} * \frac{11605}{(T+273.15)}}
$$

where $\mu$ is viscosity, $\alpha_{1-3}$ are constants, $c$ is protein concentration and $T$ is temperature $\left({ }^{\circ} \mathrm{C}\right)$.

The viscosity model is shown in Figure 3 (left). The curves represent protein concentration at nominal value and at the extremes of tolerances. The impact on injection time is depicted in Figure 3 (right). Maximum variation in viscosity (5.9-12.9 $\mathrm{mPa} \cdot \mathrm{s})$ can double the injection time $(2.1-4.0 \mathrm{~s})$. The accuracy of the viscosity prediction is within $5 \%$ of the nominal value (of the available data).

\section{Stopper friction model}

The simplest way to model friction is to use a friction coefficient in combination with a normal force which is a valid approach for dry friction. ${ }^{7}$ The inner wall of the syringe contained in the autoinjector, however, is siliconized; that is, the syringe barrel-to-stopper contact surface is lubricated. Therefore, the stopper friction model has to account for wet friction which is dependent on velocity. ${ }^{1}$
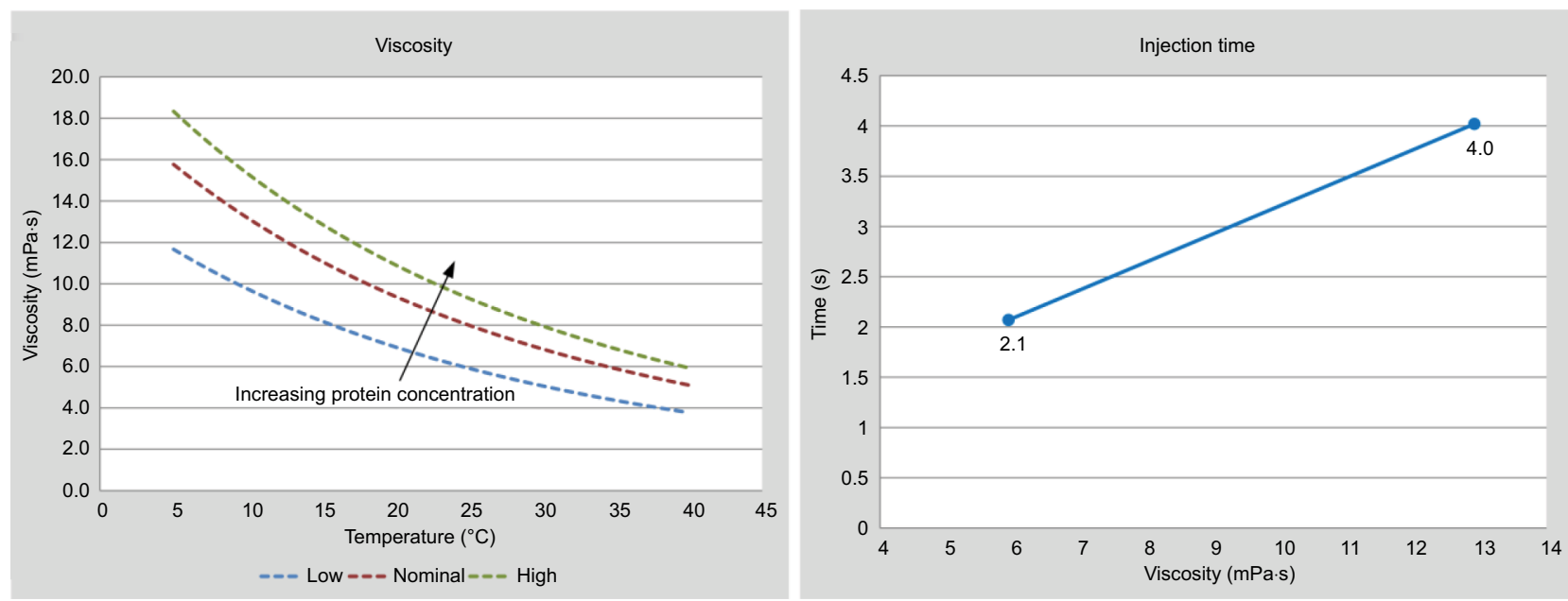

Figure 3 Viscosity model (left) and impact of viscosity variability on injection time (right).

Notes: Viscosity is modeled as a function of protein concentration and temperature. Low viscosity (low protein concentration, $\left.25^{\circ} \mathrm{C}\right)$ leads to short injection time $(2.1 \mathrm{~s})$, while high viscosity (high protein concentration, $15^{\circ} \mathrm{C}$ ) leads to long injection time $(4.0 \mathrm{~s})$. 


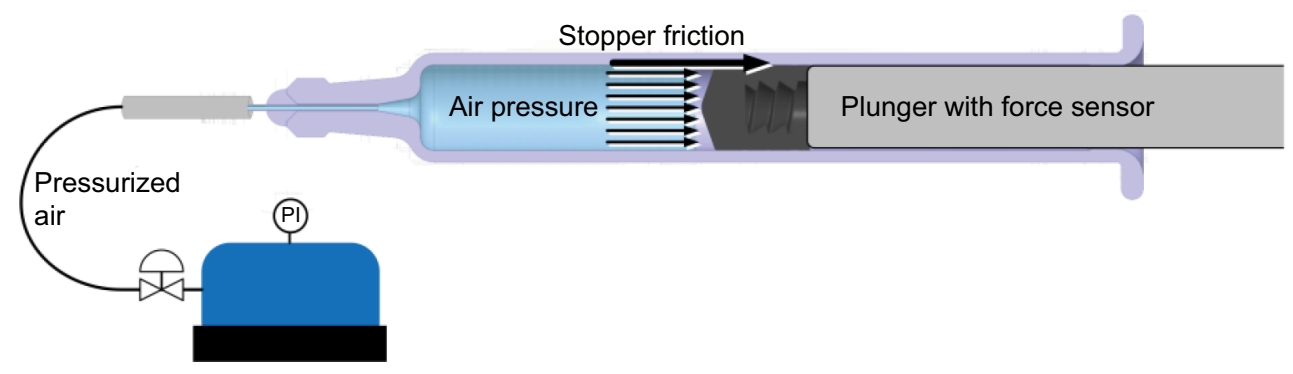

Figure 4 Experimental setup for friction force measurements by mimicking fluidic force by means of pressurized air connected at the needle end.

Investigations have shown that stopper friction is also dependent on the fluidic force that counters the stopper movement. Compression of the stopper leads to a radial deformation which increases the normal force at the syringe barrel-to-stopper contact surface and eventually results in higher stopper friction.

Stopper friction was measured in empty syringes. A special setup was required to mimic the fluidic force. For this purpose, the syringes were connected to an air compression unit at the needle end (Figure 4). Because of the low viscosity of air, the pressure drop in the needle is negligible, and the measured stopper friction is a result of velocity and stopper deformation only.

Measurements were performed over the whole range of expected stopper velocities and over the full pressure range representative of the fluidic pressure during drug delivery. A high degree of linearity was found for stopper friction as a function of velocity at different pressure levels (Figure 5, left). Therefore, the stopper friction force at each simulation step can be determined with sufficient accuracy by means of a two-step linear interpolation (Figure 5, right). In a first step, the force value for a given velocity is calculated along both pressure curves. In a second step, interpolation between these two force values yields the force at the current pressure level.

Figure 6 compares the three above-mentioned options for modeling stopper friction and the impact on simulated injection time. The curves show the different force values the models calculate over the course of an injection. By simplifying the model, injection times deviate from the nominal value $(3.0 \mathrm{~s})$ by $10 \%(2.7 \mathrm{~s})$ if the fluidic force is neglected and by $17 \%(2.5 \mathrm{~s})$ if the constant value model is applied.

\section{Plunger force model}

The autoinjector is driven by a spring which is the input force to the injection time model. ${ }^{1,3}$ The usual approach to model the spring by its spring rate and compression yielded inaccurate results (injection time error $\sim 15 \%$ ). A closer look at the mechanism revealed two elements that needed to be considered in the model: First, the fingers of the component called "actuator" retain the plunger with a tight grip which introduces friction (Figure 7, a). This leads to a significant reduction of the plunger force which is the net force available for drug delivery and which is considerably smaller than the spring force. ${ }^{4}$ Second, the plunger is released from the "actuator" toward the end of drug delivery (Figure 7, b); that is, the main contributor to internal friction disappears, and the plunger force climbs to a significantly higher level for the remaining part of the drug delivery process (Figure 7, c).

\section{Validation approach}

In order to determine the accuracy of the injection time model, a validation was performed. In a first step, the injection time of specific device configurations was measured (reference data). The tested devices were disassembled, and the parameters, previously identified as main contributors to injection time in the sensitivity analysis, were determined. In a last step, these measured values were fed into the model to generate simulation data for comparison against the injection time reference data.

Ideally, a validation would include all contributing parameters at their extremes, for example, minimum and maximum specified needle diameter. However, the nature of most device components makes it almost impossible to source these components at the extremes of tolerances, for example, injection molded parts or glass syringes.

The validation focused on parameters that could be set across the whole specified range, namely protein concentration and temperature. Surrogates were produced with defined viscosities to mimic specific protein concentration and temperature combinations. Two springs with two different spring rates were used to mimic spring contribution. Other parameters the dimensions of which could not be set at extremes of tolerance were measured and also used as inputs to the validation model. 

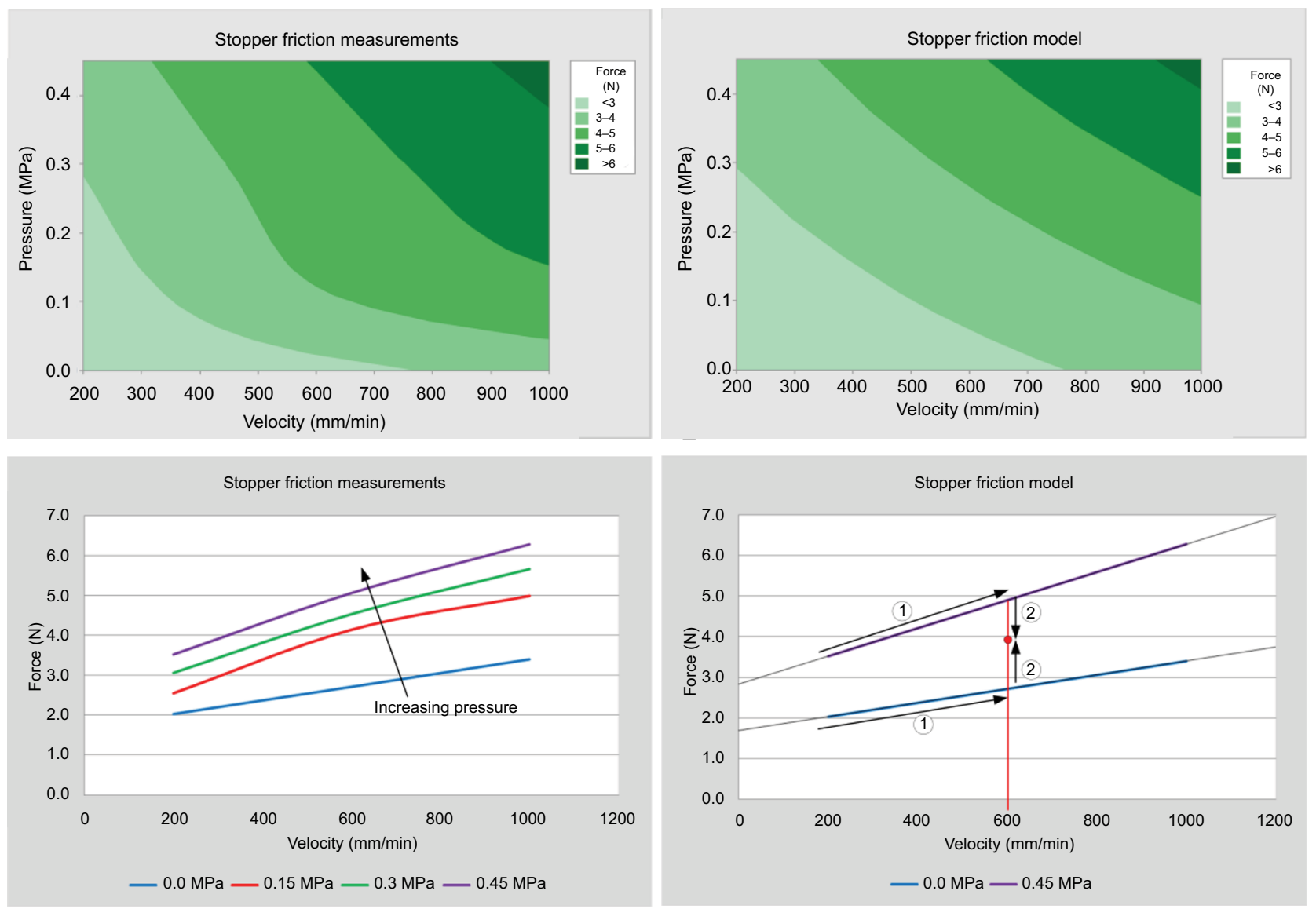

Figure 5 Stopper friction as a function of velocity and fluidic pressure: measurements ( $\mathrm{n}=30$ per data point) (left); model for use with a two-step linear interpolation (right). Note: Arrows and numbers indicate direction and sequence of the two-step interpolation.

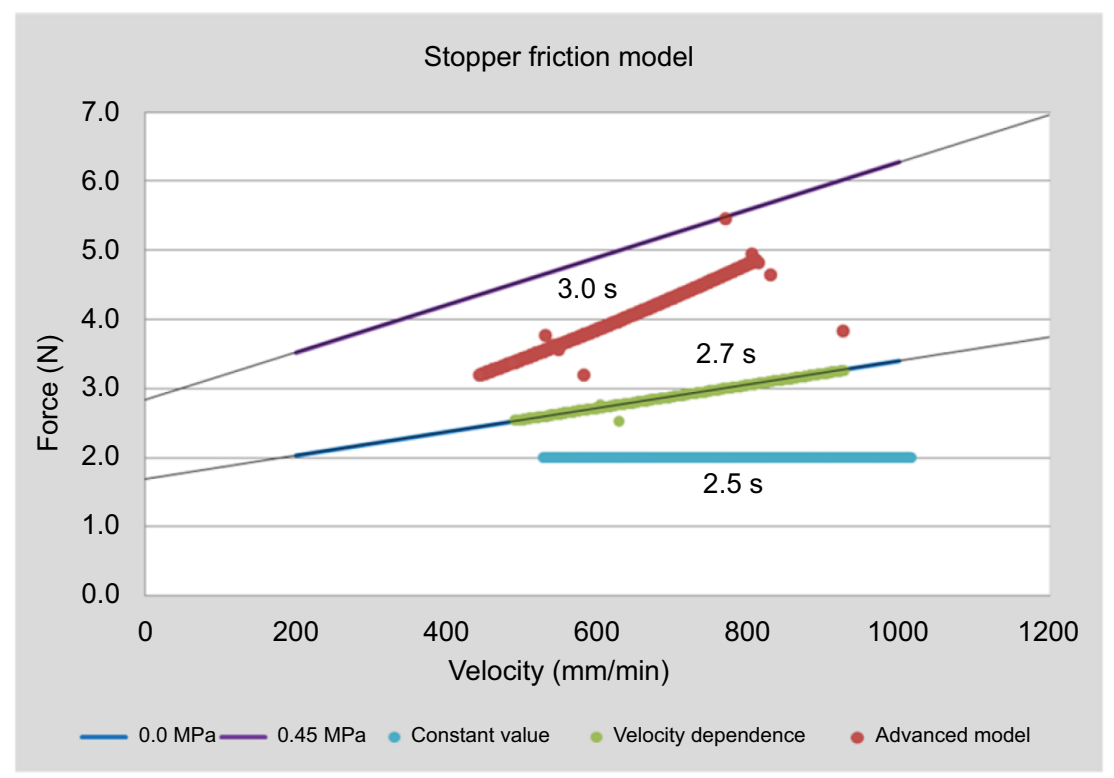

Figure 6 Comparison of friction force models with regard to impact on injection time.

Notes: Stopper friction dependent on velocity and fluidic force leads to an injection time of $3.0 \mathrm{~s}$. By only considering dependency on velocity, injection time decreases to $2.7 \mathrm{~s}$. If friction is assumed to be constant (friction force value experimentally determined for a $10 \mathrm{~s}$ injection), injection time further decreases to $2.5 \mathrm{~s}$. 


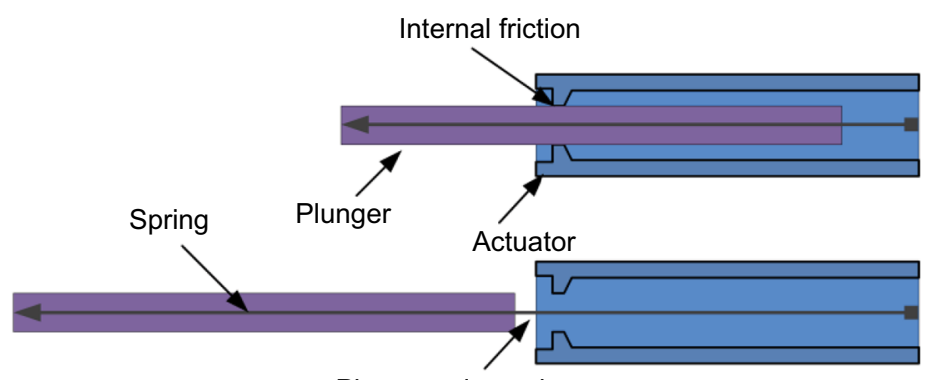

(a)

Plunger released

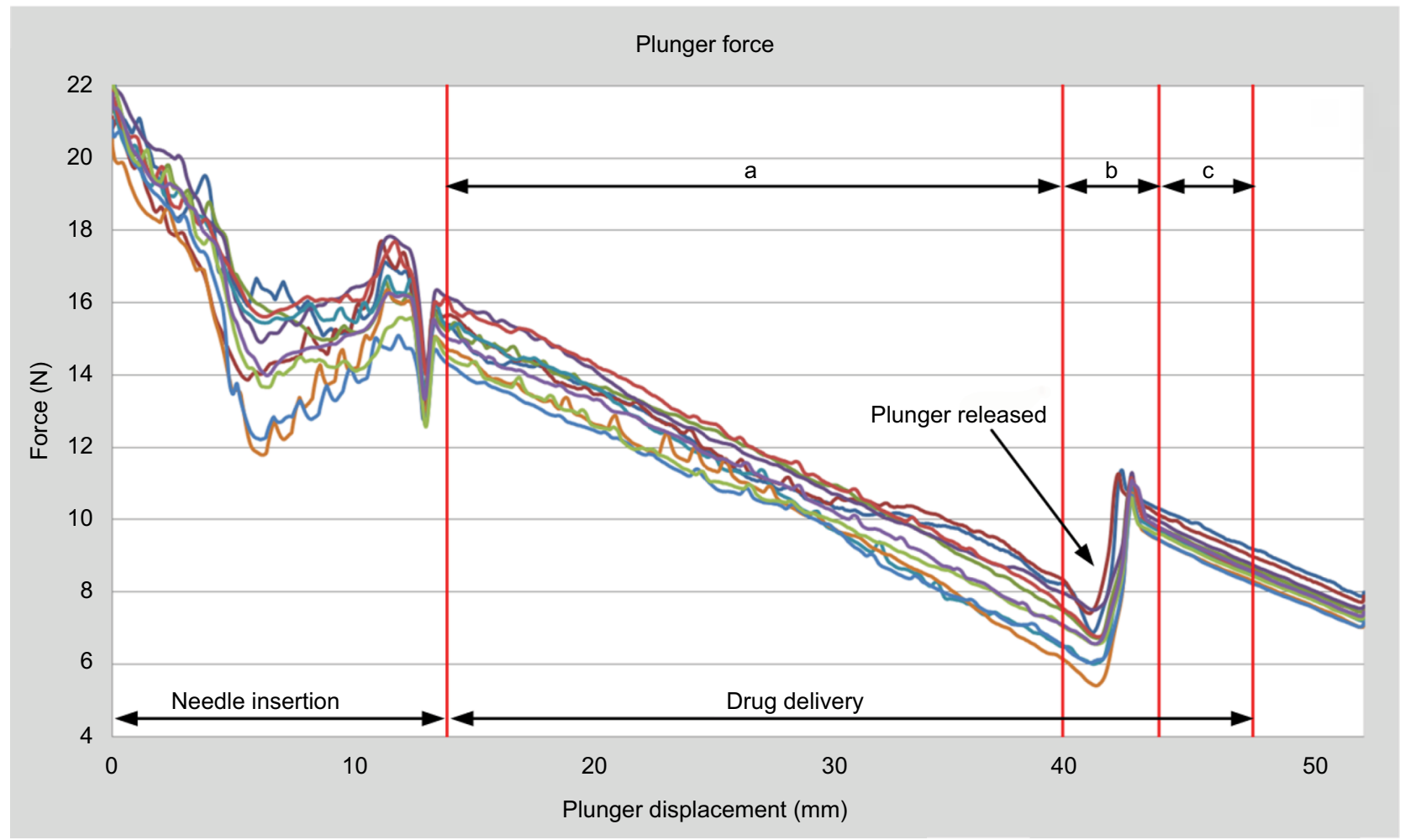

Figure 7 Components of the autoinjector's drive system shown in two states: before and after release of the plunger (top); plunger force measurements showing different levels of friction (bottom).

Notes: In the first section of the drug delivery process, the plunger is retained by the "actuator" which creates significant internal friction (a). Upon release of the plunger (b), the friction disappears, and the measurements show a considerably increased plunger force (c).

Five device configurations were created for the validation study (Table 1 and Figure 1). These configurations cover the whole expected viscosity range of the $\mathrm{mAb}$ under autoinjector use conditions which is $5.9-12.9 \mathrm{mPa} \cdot \mathrm{s}$. One device configuration contained the actual mAb with a viscosity of 10.9 $\mathrm{mPa} \cdot \mathrm{s}$ at the tested temperature, and one device configuration with the low viscosity surrogate contained a weaker spring.

\section{Results and discussion Sensitivity analysis}

A sensitivity analysis was performed to identify the main contributors to injection time. The overall sensitivity of injection time to an input parameter is a combination of two factors: the model sensitivity of injection time to the input parameter and the input parameter's uncertainty.

The percentage contributions of the five main parameters are shown in Figure 8. This result confirms the necessity for advanced models for viscosity (as a function of protein concentration and liquid temperature), plunger force and stopper friction in order to reduce the modeling error because these parameters have the biggest impact on injection time. Further, this information is pivotal for the validation study, as the modeling error can only be minimized if these input parameters can be experimentally determined with sufficient accuracy. 
Table I Overview of device configurations used for the model validation

\begin{tabular}{llll}
\hline Viscosity & PFS content & Spring rate & Needle \\
\hline $5.9 \mathrm{mPa} \cdot \mathrm{s}$ & Surrogate & Regular & $26 \mathrm{G} \mathrm{TW}$ \\
$5.9 \mathrm{mPa} \cdot \mathrm{s}$ & Surrogate & Low & $26 \mathrm{G} \mathrm{TW}$ \\
$8.9 \mathrm{mPa} \cdot \mathrm{s}$ & Surrogate & Regular & $26 \mathrm{G} \mathrm{TW}$ \\
$10.9 \mathrm{mPa} \cdot \mathrm{s}$ & $\mathrm{mAb}$ & Regular & $26 \mathrm{G} \mathrm{TW}$ \\
$12.9 \mathrm{mPa} \cdot \mathrm{s}$ & Surrogate & Regular & $26 \mathrm{G} \mathrm{TW}$ \\
\hline
\end{tabular}

Notes: Surrogates were produced to match the mAb viscosity for different protein concentration and temperature combinations. For testing, the autoinjectors were preconditioned to the specified temperatures and used immediately after removal from the climate chamber in order to ensure the corresponding viscosity.

Abbreviations: PFS, pre-filled syringe; mAb, monoclonal antibody drug.

\section{Model validation}

When assessing the validity of a mathematical model, it is important to understand the model prediction error. The equations used are not inclusive of all contributing variables that may influence injection time. Model errors may arise from numerous sources, including unaccounted friction, component geometry (eg, deflection, distortion) and assumed boundary conditions. Introducing a correction factor to compensate for discrepancies between modeled and real-world data is a widely used approach in mathematical modeling. The model presented in this article does not apply any correction factor and achieves an error range of less than $6 \%$ across the five device configurations tested.

The outcome of the validation study is provided in Figure 9. A good correlation of measured and modeled injection times is shown, with the model slightly overestimating injection time, as depicted in Figure 9 (left). The modeling error for all five device configurations, as shown in Figure 9 (right), is smaller than $12 \%$, and the error range is less than $6 \%$. The consistent overestimation of injection time suggests a small bias in the model which could be accounted for by reducing internal friction. An increase in modeling error as a consequence of increasing viscosity, and thus increasing injection time, cannot be confirmed.

Additional investigations to assess the robustness of the model have shown a trend for overestimation beyond $15 \%$ if the combination of the main contributing factors (viscosity, needle diameter and plunger force) resulted in noticeably longer injection times ( $>12$ seconds). It was found that in such cases stopper velocity was outside the model range where the linear model might not apply. Further consideration is required for formulations with water-like viscosities which can be delivered in very short times. Assuming laminar flow through the needle, and therefore applying the Hagen-Poiseuille formula, might not be appropriate in such cases.

\section{Component variability}

In order to assess the impact of component variability, a Monte Carlo simulation was run with all input parameters following a defined statistical distribution. The histogram for injection time based on variation of all system parameters in 10,000 trials is provided in Figure 10.

Comparing the range of simulated injection times from the validation study $(2.4-4.8 \mathrm{~s})$ with the probability

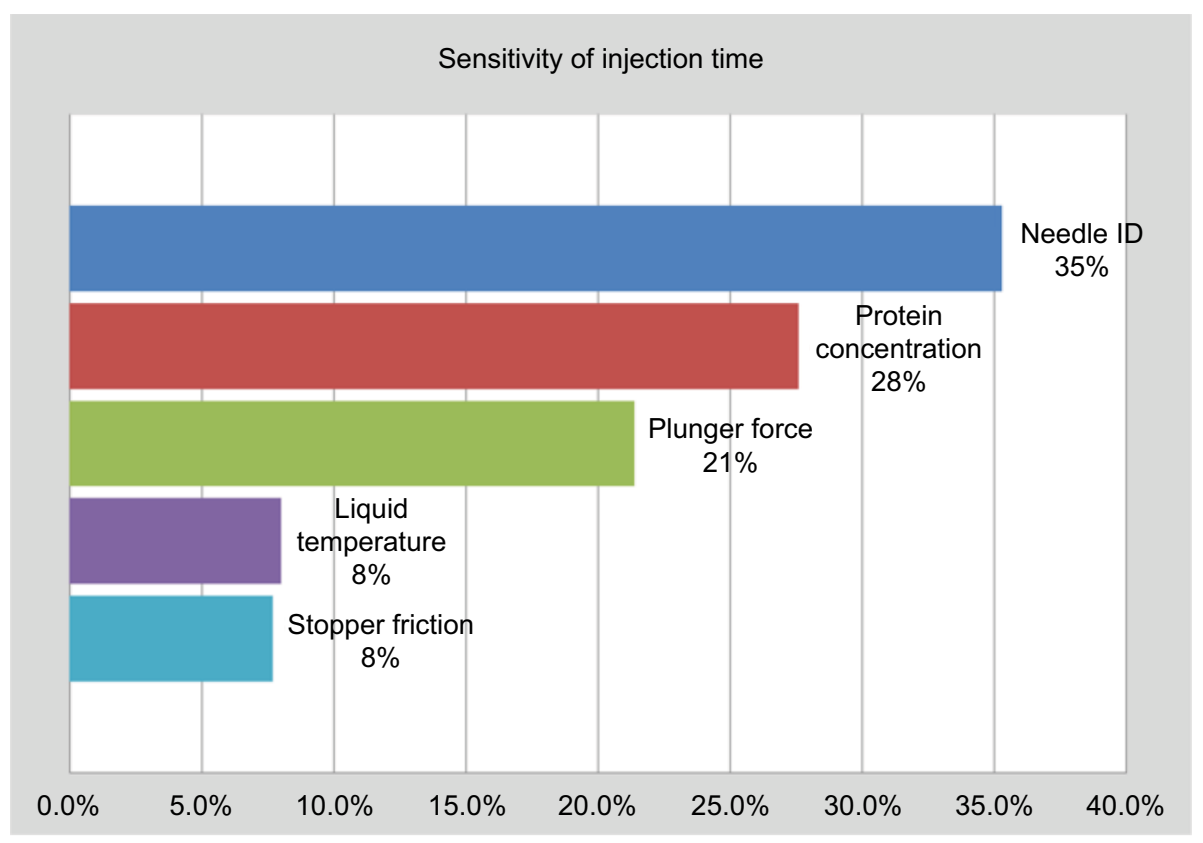

Figure 8 Sensitivity analysis showing the five main contributors to injection time. 

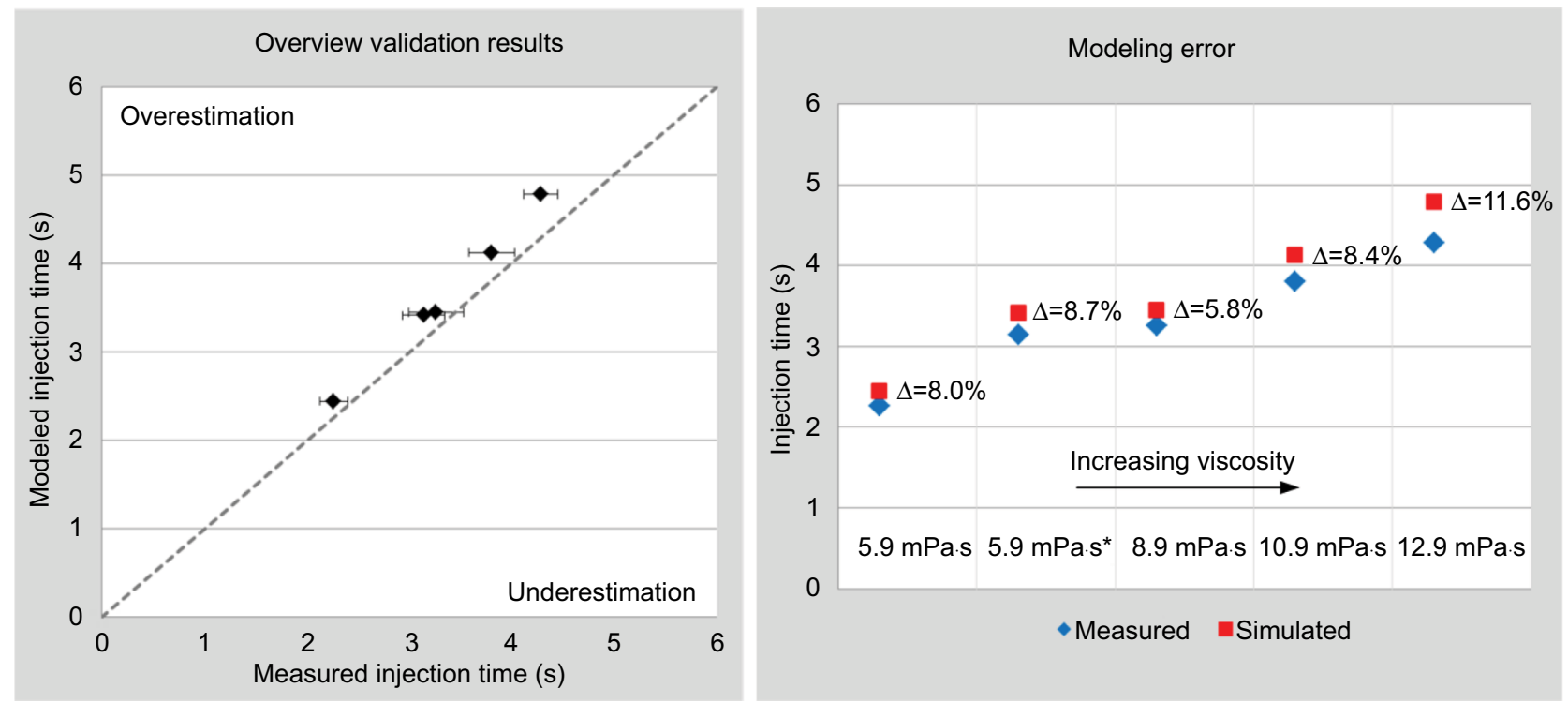

Figure 9 Validation results showing general agreement between simulations and measurements (left) and modeling error (right) for the different device configurations. Notes: The device configurations are sorted by increasing viscosity. The asterisk $\left(^{*}\right)$ indicates a device with a weaker spring which leads to a longer injection time with the same viscosity. The configuration with the $10.9 \mathrm{mPa} \cdot \mathrm{s}$ viscosity contained the actual mAb.

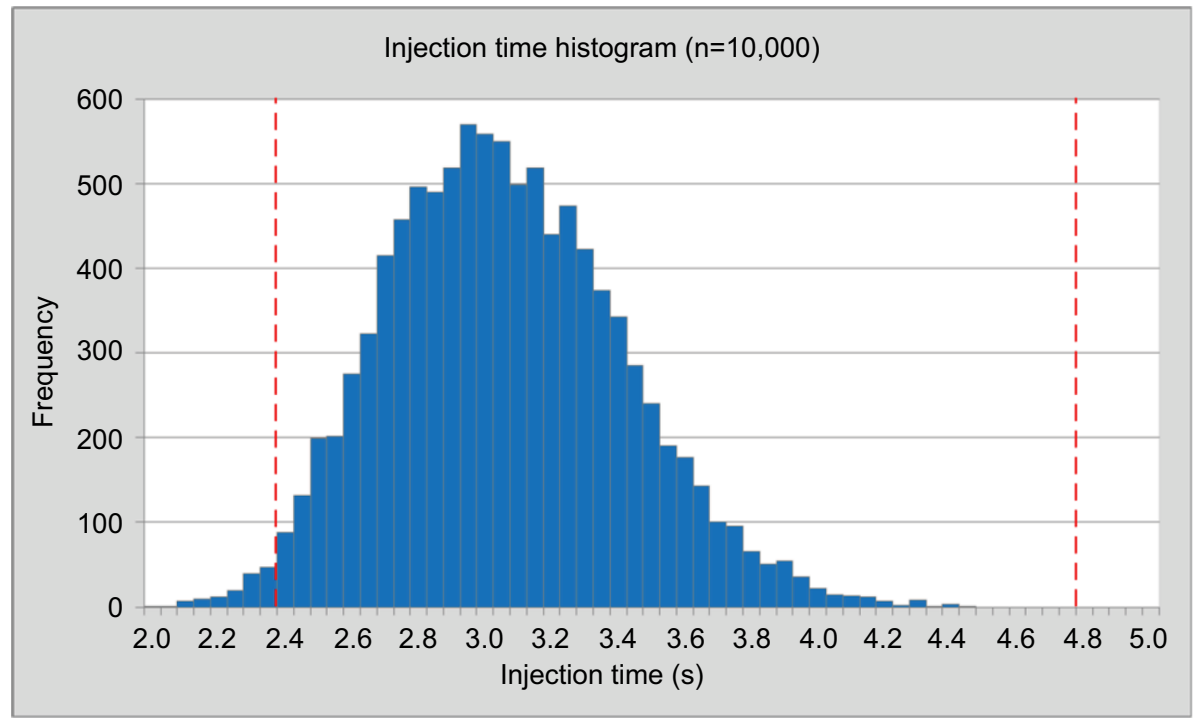

Figure 10 Histogram showing the range of expected injection times based on variation of all components within the specified tolerance bands. Dotted lines indicate the range of injection times in the validation study.

distribution in Figure 10, it can be seen that the validation study spans a significant portion of the expected injection times. This is important because the histogram includes variation of all input parameters across their whole tolerance band while the number of varied parameters in the validation study was limited. This confirms the actual impact of a viscosity change as found in the sensitivity analysis. The shift of the validation results toward longer injection times can be traced back to the plunger force in the tested devices which was at the lower end of the specification.

\section{Quality of input data}

The quality of input data is highly relevant for the validity of the simulation results as low-quality input data (eg, due to inaccurate measurements) may introduce large variation in the predicted injection times. Simulation results associated with a high uncertainty have little value for decision making in the development process and may even lead to wrong conclusions.

The generation of input data for stopper friction is inherently challenging, and the problem of considerable variation occurring in stopper friction measurements has been 
addressed before. ${ }^{1}$ Figure 11 shows the friction force measurements ( $\mathrm{n}=15$ ) at $200 \mathrm{~mm} / \mathrm{min}$ (left) and $1000 \mathrm{~mm} / \mathrm{min}$ (right). The standard deviations amount to $11 \%$ and $14 \%$, respectively, and the measurement ranges amount to $45 \%$ and $53 \%$ of the mean value. These data confirm the large variation and also suggest that input data quality for friction force decreases with increasing test speeds which are, however, needed to reflect conditions in the autoinjector during drug delivery.

Friction force measurements cannot be performed on the same samples as injection time due to the destructive nature of both test methods. Therefore, friction force was measured on a representative set of syringes (same batch), and the same data set based on averaged values was used in all simulations. As a consequence, the large variation seen in the friction force measurements is not translated into the simulations. This is confirmed by the outcome of the validation study which shows considerably less variation.

\section{Understanding the design space}

During development and optimization of an autoinjector, it is important to know the design space and to understand the interactions in the system. The following example is used to demonstrate how the injection time model enables engineers to explore the potential of adjusting the plunger force in order to meet a target injection time.

It can be seen in Equation 2 that there exists a reciprocal relationship between injection time and plunger force. The corresponding graph, a hyperbola, is shown in Figure 12. It is evident that an increase in plunger force by a defined factor does not lead to a decrease in injection time by the same
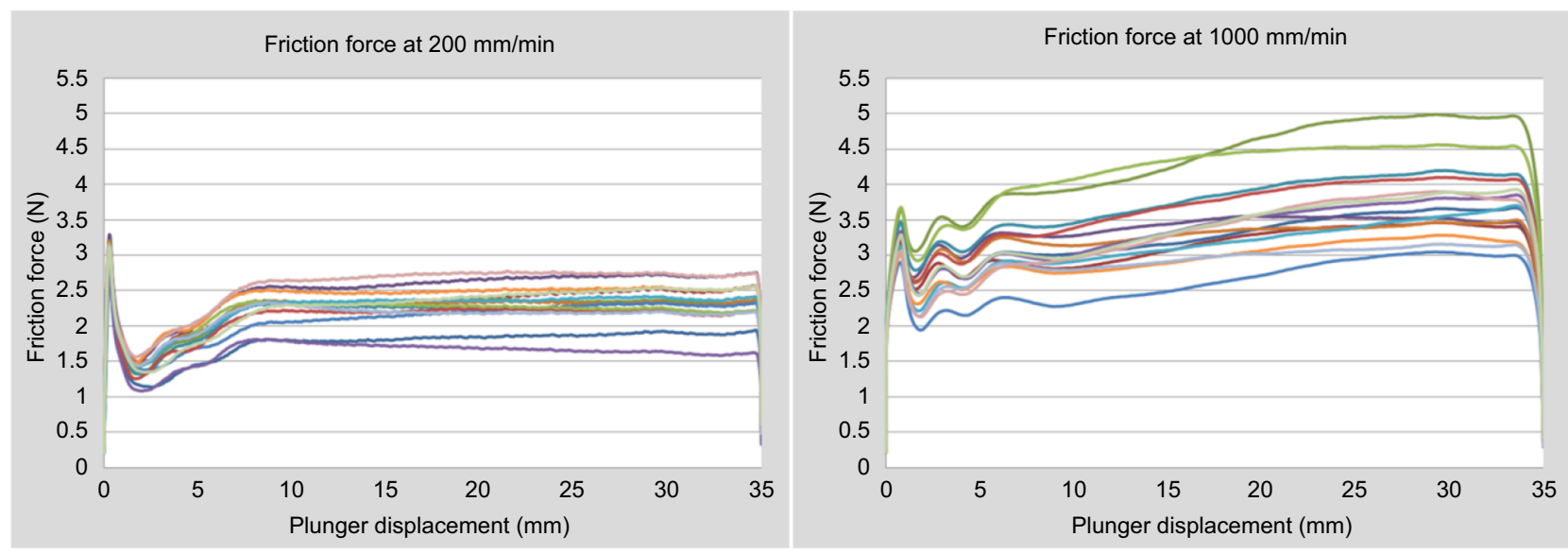

Figure I I Stopper friction measurements at $200 \mathrm{~mm} / \mathrm{min}$ (left) and $1000 \mathrm{~mm} / \mathrm{min}$ (right) showing considerable variation which seems to increase with test speed.

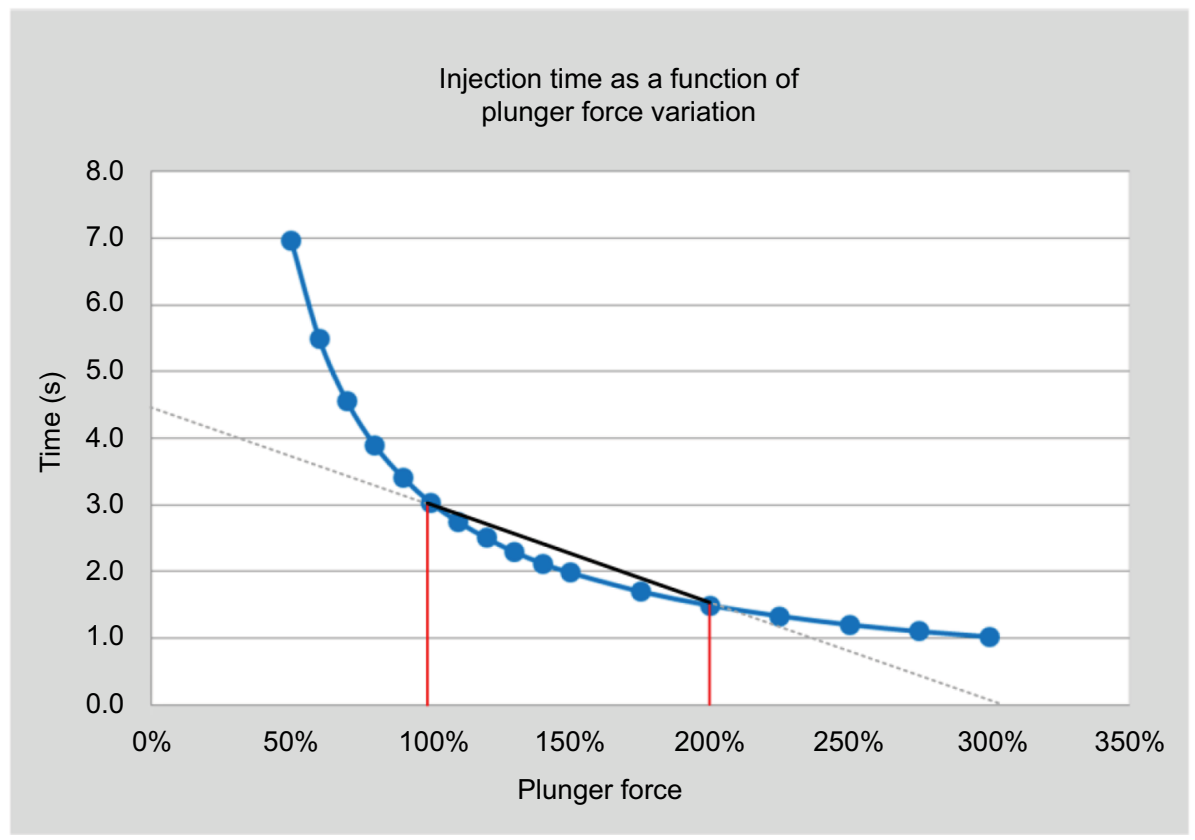

Figure 12 Displaying injection time as a function of plunger force variation helps to anticipate the impact of a force change on injection time. 
factor as the relationship is nonlinear. In this example, doubling the plunger force happens to reduce the injection time by half. However, a further increase of the plunger force to three times its initial value would only have a marginal effect. This example nicely shows the limits of the design space and the challenges with nonlinear relationships in the system.

\section{Conclusion}

An injection time model for an autoinjector was discussed in this article. Previously published works were significantly extended by introducing advanced models for the main contributors to injection time and by providing quantitative information about the modeling error determined through model validation. It was shown that the selected modeling approach is considerably accurate and inertial forces can be neglected without compromising the quality of the results. By considering static forces only, there is no need to deal with complicated differential equations, and therefore, solving the proposed model is computationally inexpensive which enables running comprehensive statistical simulations. The validation shows a highly satisfying accuracy of the model with a modeling error below $12 \%$ even though a large number of parameters were considered and variability of input data presented considerable challenges.

\section{Acknowledgments}

The authors would like to thank Chris Muenzer and Stefan Fischer for work on the viscosity model; Fred Bathke, Jean-
Michel Courtois and Lothar Vorgrimler for execution of experimental work; Neil Cammish and Ajay Deshmukh for expert advice on modeling.

\section{Disclosure}

The authors are all employees of F. Hoffmann-La Roche. The authors report no other conflicts of interest in this work.

\section{References}

1. Kivitz A, Segurado OG. HUMIRA pen: a novel autoinjection device for subcutaneous injection of the fully human monoclonal antibody adalimumab. Expert Rev Med Devices. 2007;4(2):109-116.

2. Rathore N, Pranay P, Eu B, Ji W, Walls E. Variability in syringe components and its impact on functionality of delivery systems. PDA J Pharm Sci Technol. 2011;65(5):468-480.

3. Wilkins J, Simpson I. Mathematical modeling for faster autoinjector design. Drug Dev Deliv. 2012;12(6):41-45.

4. Ajaghari N, Authelin JR. Modeling of autoinjectors. Presented at: PDA Parenteral Packaging; April 13, 2016; Venice.

5. Allmendinger A, Fischer S, Huwyler J, et al. Rheological characterization and injection forces of concentrated protein formulations: an alternative predictive model for non-Newtonian solutions. Eur J Pharm Biopharm. 2014;87(2):318-328.

6. Rathore N, Pranay P, Bernacki J, Eu B, Ji W, Walls E. Characterization of protein rheology and delivery forces for combination products. J Pharm Sci. 2012;101(12):4472-4480.

7. Lange J, Urbanek L, Burren S. Development of devices for self-injection: using tribological analysis to optimize injection force. Med Devices (Auckl). 2016;9:93-103.

8. Fischer I, Schmidt A, Bryant A, Besheer A. Calculation of injection forces for highly concentrated protein solutions. Int J Pharm. 2015;493(1-2):70-74.
Medical Devices: Evidence and Research

\section{Publish your work in this journal}

Medical Devices: Evidence and Research is an international, peerreviewed, open access journal that focuses on the evidence, technology, research, and expert opinion supporting the use and application of medical devices in the diagnosis, monitoring, treatment and management of clinical conditions and physiological processes. The identification of novel

\section{Dovepress}

devices and optimal use of existing devices which will lead to improved clinical outcomes and more effective patient management and safety is a key feature. The manuscript management system is completely online and includes a quick and fair peer-review system. Visit http://www. dovepress.com/testimonials.php to read real quotes from authors. 